\title{
GI-MS46-P07 | Facilities for Macromolecular Crystallography at the HZB
}

Gerlach, Martin (Helmholtz-Zentrum Berlin, Berlin, GER); Feiler, Christian (Helmholtz-Zentrum Berlin, Berlin, GER); Förster, Ronald (Helmholtz-Zentrum Berlin, Berlin, GER); Gless, Christine (Helmholtz-Zentrum Berlin, Berlin, GER); Hauß, Thomas (Helmholtz-Zentrum Berlin, Berlin, GER); He, Huiling (Helmholtz-Zentrum Berlin, Berlin, GER); Hellmig, Michael (HelmholtzZentrum Berlin, Berlin, GER); Kastner, Alexandra (Helmholtz-Zentrum Berlin, Berlin, GER); Steffien, Michael (Helmholtz-Zentrum Berlin, Berlin, GER); Taberman, Helena (Helmholtz-Zentrum Berlin, Berlin, GER); Wollenhaupt, Jan (Helmholtz-Zentrum Berlin, Berlin, GER); Weiss, Manfred S. (Helmholtz-Zentrum Berlin, Berlin, GER)

The Helmholtz-Zentrum Berlin (HZB) operates three beamlines for macromolecular crystallography (MX) at the electron storage ring BESSY II [1,2,3]. BL14.1 and BL14.2 are tunable in the photon energy range from 5 to $16 \mathrm{keV}$, while BL14.3 is a fixed-energy side station (13.8 keV). They feature state-of-the-art experimental stations and ancillary facilities, serving more than 100 research groups across Europe. With almost 3000 protein structures measured at BESSY II that have resulted in protein data base (PDB) depositions so far, they are the most productive MX-stations in Germany.

The experimental endstation of BL14.1 provides high degree of automation and is equipped with a PILATUS 6M detector, a CATS sample changer robot and an MD2 multi-axis goniometer. BL14.2 features a PILATUS3 S 2M detector, a G-Rob sample changer and a piezo-controlled nanodiffractometer. Its sample dewar can accommodate up to 294 samples, supporting both, SPINE- and UNIPUCK standards, allowing for high sample-throughput data collection. BL14.3 is equipped with a fast MD2-S microdiffractometer, including a mini-kappa axis and a plate manipulator option for in situ-crystal screening, supporting several types of 96-well plates. Furthermore, an HCLab dehydration device is available that can be used in combination with a REX rapid nozzle exchanger to freeze samples in a defined hydration state within less than a second.

[1] U. Mueller et al., J. Synch. Rad. 19, (2012), 442

[2] U. Mueller et al., Eur. Phys. J. Plus 130, (2015), 141

[3] M. Gerlach et al., Journal of large-scale research facilities 2, (2016), A47 